\title{
An embedding theorem for fields
}

\section{J.W.S. Cassels}

It is shown that every finitely generated field $K$ of characteristic 0 may be embedded in infinitely many p-adic fields in such a way that the images of any given finite set $C$ of non-zero elements of $K$ are p-adic units. The result is suggested by Lech's proof of his generalization of Mahler's theorem on recurrent sequences. It also provides a simple proof of Selberg's theorem about torsion-free normal subgroups of matrix groups.

THEOREM I. Let $K$ be a finitely generated extension of the rational field $Q$ and let $C$ be a finite set of non-zero elements of $K$. Then there exist infinitely many primes $p$ such that there is an embedding

$$
\alpha: K \rightarrow Q_{p}
$$

of $K$ into the p-adic numbers $Q_{p}$ for which

$$
|\alpha c|=1 \text { (aIz } c \in C) \text {. }
$$

Here || denotes the p-adic valuation.

This theorem does not appear to have been stated explicitly before. The paper of Lech [3] contains implicitly a weaker form in which $Q_{p}$ is replaced by some algebraic extension of a p-adic field.

Lech uses his result to generalize Mahler's theorem [4] about the values taken by recurrent sequences to any field of characteristic 0 . Indeed Mahler's proof works in a p-adic field and so the generalization is an immediate consequence of the original theorem and the embedding.

Received 21 November 1975. 
Another application is:

THEOREM II (Selberg [6]; see also Borel [1]). Let $G$ be a finitely generated group of matrices in a field $k$ of characteristic 0 . Then $G$ contains a normal torsion-free subgroup of finite index.

Proof. We can take for $C$ the set of non-zero elements of $A, A^{-1}$, where $A$ runs through a set of generators of $G$, and for $K$ the subfield of $k$ generated by $C$. We can also suppose that $p \neq 2$. If $\alpha$ is as given by Theorem $I$, the elements of the matrices in $\alpha G$ are all in the p-adic integers $Z_{p}$. The subgroup of $\alpha G$ consisting of the matrices of the type $I+p B$, where $B$ has elements in $Z_{p}$, is clearly normal and is torsion-free. [For we have to show that $(I+p B)^{n} \neq I$ whenever $B \neq 0$ and it is enough to show this when $n$ is a prime. But then

$(I+p B)^{n}=I+n p B+\ldots+p^{n} B^{n}$ and the largest element of $n p B$ is $p$-adically greater than the elements of the subsequent terms. The condition $p \neq 2$ is needed when $n=p$.]

The proof given below of Theorem I follows Lech's argument quite closely. There is an additional twist, but that is also familiar from other contexts. We require three simple lemmas.

LEMMA 1. Let

$$
\begin{aligned}
f_{j}\left(X_{1}, \ldots, X_{n}\right) & \in Z\left[X_{1}, \ldots, x_{n}\right] \quad(1 \leq j \leq J) \\
& \neq 0
\end{aligned}
$$

be a finite set of non-zero polynomials in the indeterminates $x_{1}, \ldots, x_{n}$ with rational integral coefficients. Then there are rational integers $a_{1}, \ldots, a_{n}$ such that

$$
f_{j}\left(a_{1}, \ldots, a_{n}\right) \neq 0 \quad(1 \leq j \leq J)
$$

Proof. If $n=1$ pick $a_{1}$ distinct from the finitely many roots of the $f_{j}$. If $n>1$ use induction to pick $a_{2}, \ldots, a_{n}$ so that $f_{j}\left(x_{1}, a_{2}, \ldots, a_{n}\right) \neq 0$ and then pick $a_{1}$.

LEMMA 2. Let $g(X) \in Z[X]$ be a non-constant polynomial in the single indeterminate $X$ with rational integral coefficients. Then there 
are infinitely many primes $p$ for which there is a solution $b \in Z$ of the congruence

$$
g(b) \equiv 0 \quad(\bmod p)
$$

Proof. Let $\beta$ be a root of $g(\beta)=0$. Then it is enough to show that there are infinitely many first-degree primes in $Q(\beta)$; and this follows from elementary analytic number-theory. (See, for example, Borevich and Shafarevich [2], Chapter V, \$3.1.)

LEMMA 3. $Q_{p}$ has infinite transcendence degree over $Q$.

Proof. For $Q_{p}$ is uncountable but the algebraic closure of any extension of $Q$ of finite transcendence degree is countable.

Proof of Theorem I. We note first that, by taking a larger set for $C$ if necessary, we may suppose that $c^{-1} \in C$ whenever $c \in C$. It will thus be enough to find primes $p$ and embeddings $\alpha$ for which

$$
|\alpha c| \leq 1 \quad(\text { all } \quad c \in C) \text {. }
$$

Let $x_{1}, \ldots, x_{m}(m \geq 0)$ be a transcendence base of $K$ over $Q$. Then $x_{1}, \ldots, x_{m}$ are independent transcendentals and

$$
K=Q\left(y, x_{1}, \ldots, x_{m}\right)
$$

for some $y \in K$ which is algebraic over $Q\left(x_{1}, \ldots, x_{m}\right)$. We can thus put each $c \in C$ into the shape.

$$
c=U_{c}\left(y, x_{1}, \ldots, x_{m}\right) / V_{c}\left(x_{1}, \ldots, x_{m}\right)
$$

where

$$
\left\{\begin{aligned}
U_{c}\left(Y, X_{1}, \ldots, X_{m}\right) & \in Z\left[Y, X_{1}, \ldots, X_{m}\right] \\
\text { and } & \\
V_{c}\left(X_{1}, \ldots, X_{m}\right) & \in Z\left[X_{1}, \ldots, x_{m}\right] \\
& \neq 0
\end{aligned}\right.
$$

Here $Z$ denotes the rational integers and $Y, X_{1}, \ldots, X_{m}$ are indeterminates.

We can select an irreducible equation $G(Y)=0$ for $y$ over 
$Q\left(x_{1}, \ldots, x_{m}\right)$ of the shape

$$
G(Y)=H\left(Y, x_{1}, \ldots, x_{m}\right)
$$

where

$$
H\left(Y, X_{1}, \ldots, X_{m}\right) \in Z\left[Y, X_{1}, \ldots, X_{m}\right]
$$

If $H$ is of degree $s$ in $Y$ we denote the coefficient of $Y^{s}$ by $H_{0}\left(x_{1}, \ldots, x_{m}\right)$, so

$$
\begin{aligned}
H_{0}\left(x_{1}, \ldots, x_{m}\right) & \in Z\left[x_{1}, \ldots, x_{m}\right] \\
& \neq 0 .
\end{aligned}
$$

The discriminant of $G(y)$ is of the shape $\Delta\left(x_{1}, \ldots, x_{m}\right)$, where

$$
\begin{aligned}
\Delta\left(x_{1}, \ldots, x_{m}\right) & \in \mathrm{Z}\left[x_{1}, \ldots, x_{m}\right] \\
& \neq 0 .
\end{aligned}
$$

By Lemma 1 we can pick $a_{1}, \ldots, a_{m} \in Z$ such that

$$
\begin{aligned}
& \Delta\left(a_{1}, \ldots, a_{m}\right) \neq 0, \\
& H_{0}\left(a_{1}, \ldots, a_{m}\right) \neq 0, \\
& \left.V_{c}\left(a_{1}, \ldots, a_{m}\right) \neq 0 \text { (all } c \in C\right) .
\end{aligned}
$$

By (4) and Lemma 2 there are infinitely many primes $p \neq 2$ for which there is a solution $b \in Z$ of the congruence

$$
H\left(b, a_{1}, \ldots, a_{m}\right) \equiv 0(\bmod p) .
$$

On excluding finitely many of these primes we may also suppose by (3), (4), (5), that

$$
\begin{aligned}
& \Delta\left(a_{1}, \ldots, a_{m}\right) \neq 0(\bmod p), \\
& u_{0}\left(a_{1}, \ldots, a_{m}\right) \neq 0(\bmod p), \\
& V_{c}\left(a_{1}, \ldots, a_{m}\right) \neq 0(\bmod p) \quad(\text { all } c \in C) .
\end{aligned}
$$

By Lemma 3 we can select $m$ independent transcendentals $\theta_{1}, \ldots, \theta_{m}$ in $Q_{p}$. On replacing the $\theta_{j}$ by $p^{t} \theta_{j}$ with large positive integral $t$ 
if necessary, we may suppose that

$$
\left|\theta_{j}\right|<1 \quad(1 \leq j \leq m) \text {. }
$$

Then

$$
\xi_{j}=a_{j}+\theta_{j} \quad(1 \leq j \leq m)
$$

is a set of independent transcendentals in $Q_{p}$ with

$$
\left|\xi_{j}-a_{j}\right|<1
$$

Now by $(6),(7),(10)$ and Hensel's Lemma there is an $n \in Q_{p}$ with $|n-b|<1$ and

$$
H\left(\eta, \xi_{1}, \ldots, \xi_{m}\right)=0
$$

Thus

$$
\begin{aligned}
& x_{j} \rightarrow \xi_{j} \quad(1 \leq j \leq m), \\
& y \rightarrow n
\end{aligned}
$$

defines an isomorphism $\alpha$ of $K=Q\left(y, x_{1}, \ldots, x_{m}\right)$ with $Q\left(n, \xi_{1}, \ldots, \xi_{m}\right) \subset Q_{p}$.

Further,

$$
\left|U_{c}\left(\eta, \xi_{1}, \ldots, \xi_{m}\right)\right| \leq 1,\left|V_{c}\left(\xi_{1}, \ldots, \xi_{m}\right)\right| \leq 1
$$

by (2) and since $\left|\xi_{j}\right| \leq 1,|\eta| \leq 1 ;$ and indeed

$$
\left|v_{c}\left(\xi_{1}, \ldots, \xi_{m}\right)\right|=1
$$

by (9) and (10). Hence

$$
\begin{aligned}
|\alpha c| & =\left|U_{c}\left(n, \xi_{1}, \ldots, \xi_{m}\right)\right| /\left|v_{c}\left(\xi_{1}, \ldots, \xi_{m}\right)\right| \\
& \leq 1 .
\end{aligned}
$$

This completes the proof. 


\section{References}

[1] Armand Borel, "Compact Clifford-Klein forms of symmetric spaces", Topology 2 (1963), 111-122.

[2] Z.1. Borevich and I.R. Shafarevich, Number theory (translated by Newcomb Greenleaf for Scripta Technica. Pure and Applied Mathematics, 20. Academic Press, New York and London, 1966).

[3] Christer Lech, "A note on recurring series", Ark. Mat. 2 (1954), 417-421.

[4] Kurt Mahler, "Eine arithmetische Eigenschaft der Taylor-Koeffizienten rationaler Funktionen", Proc. K. Nederl. Akad. Wetensch. Amsterdam 38 (1935), 50-60.

[5] K. Mahler, "On the Taylor coefficients of rational functions", Proc. Cambridge Philos. Soc. 52 (1956), 39-48.

[6] Atle Selberg, "On discontinuous groups in higher-dimensional symmetric spaces", Contributions to function theory, 147-164 (International Colloquium on Function Theory, Bombay, 1960. Tata Institute of Fundamental Research, Bombay, 1960).

Department of Pure Mathematics and Mathematical Statistics, University of Cambridge,

Cambridge,

UK. 